\title{
Dynamical evolution of star clusters in transient spiral arms
}

\section{Michiko Fujii ${ }^{1}$ and Junichi Baba ${ }^{2}$}

${ }^{1}$ Leiden Observatory Netherlands, email: fujii@strw.leidenuniv.nl ${ }^{2}$ Tokyo Institute of Technology

\begin{abstract}
Star clusters are one of fundamental building blocks of galactic disks. They form in a potential well of spiral arms and travel in the disk. We performed N-body simulation of star clusters in stellar disk with "live" spiral arms. In this simulation, both star clusters and stellar disks are modeled as N-body systems. We found that star clusters migrated in the galactic disk in a timescale of their galactic rotation. The tidal tails spread over a few kpc, but they might be detectable if we are able to measure their velocity.
\end{abstract}

